\title{
HIGH POWER SPLITTING OF THE CYCLOTRON RESONANCE INDUCED PHOTOCONDUCTIVITY IN n-GaAs
}

\author{
M. Weispfenning, A. Bauer, and W. Prettl \\ Institut für Angewandte Physik, Universität Regensburg \\ D-8400 Regensburg, West Germany
}

Received March 13, 1989

\begin{abstract}
At high power levels a splitting of the cyclotron resonance induced far-infrared photoconductivity in n-GaAs has been observed which is not present in the optical absorption. This effect is attributed to $2 p_{-} \rightarrow 2 p_{+}$shallow donor optical transitions which are forbidden by electric dipole selection rules but may become allowed due to electric stray fields caused by ionized impurities. Due to time inversion $2 p_{-} \rightarrow 2 p_{+}$transitions spectrally coincide with $\mathrm{N}=0 \rightarrow \mathrm{N}=1$ Landau level transitions and thus affect all optical characteristics observed under cyclotron resonance conditions. In particular the optical absorption is increased and photoconductivity is reduced by accelerating the recombination of free carriers yielding different line shapes of absorption and photoconductivity even at low intensities. This intensity controlled free carrier recombination channel has a significant effect on the lifetime of electrons in the $\mathrm{N}=1$ Landau level.
\end{abstract}

\section{Introduction}

Cyclotron resonance induced photoconductivity in high purity semiconductors at low temperatures shows a highly nonlinear dependence on both the electric bias field and the exciting far-infrared light intensity [1,2]. At low temperatures practically all carriers are bound to shallow impurities yielding a very low conductance of the sample. At a critical electric field strength free carriers gain sufficient energy so that the carrier generation rate due to impact ionization of shallow impurities exceeds the capture rate for low carrier concentration resulting in a rapid increase of the current with rising electric field. In a previous study on n-GaAs epitaxial layers it was shown that cyclotron resonance lowers the critical electric field of impact ionization breakdown resulting in a threshold- 
like behaviour of the photoconductivity due to cyclotron resonance [2]. This observation proves that electrons in both the $\mathrm{N}=0$ and $\mathrm{N}=1$ Landau level contribute to impact ionization of neutral donors whereas free electrons are predominantly captured from the $\mathrm{N}=0$ Landau level by ionized donors. Therefore optical generation of electrons in the $\mathrm{N}=1$ Landau level does not change much the impact ionization rate, however, due to the depletion of the electron number in the $\mathrm{N}=0$ Landau band, the capture rate is reduced. Thus the steady state electron concentration may be maintained with lower impact ionization rate i.e. lower electric field strength.

At low temperature where the thermal generation of free carriers is very low, the photoconductive signal vanishes up to a critical optical transition probability $\left(\sigma_{C R} F\right)_{c}$ which depends on the electric bias field and the magnetic field strength. Here $\sigma_{C R}$ is the absorption cross-section of cyclotron resonance and $F$ the photon flux density. For $\sigma_{C R} F>\left(\sigma_{C R} F\right)_{c}$ the photosignal first grows linearly with rising intensity and finally saturates. This nonlinear behaviour of the cyclotron resonance induced photoconductivity has a drastic effect on the lineshape. A Lorentzian shaped absorption cross section $\sigma_{C R}$ as function of the magnetic field strength does not yield a Lorentzian shaped signal because a non-zero signal occurs only if $\sigma_{C R}(B) F>\left(\sigma_{C R} F\right)_{c}$. The threshold-like response cuts the Lorentzian at $\left(\sigma_{C R} F\right)_{c}$ and saturation further deforms the lineshape. Thermal generation of free carriers or, in the case where the photon energy is larger than the binding energy of shallow donors, photoionization of the impurities smoothens the sharp onset of photoconductivity at the threshold yielding a superlinear increase of the signal at low intensities.

In the present contribution we report on a new nonlinear effect which occurs at larger intensities than applied previously [2]. At high intensities the cyclotron resonance induced photoconductivity line shows a splitting or, using a pictorial description, a hole is burnt into the line just at the $\mathrm{N}=0 \rightarrow \mathrm{N}=1$ Landau level transition resonance. The effect depends on the sample and is more pronounced in materials containing a larger concentration of ionized impurities. The width of the splitting and the depth of the hole increase with rising intensity. The total photoconductivity, the cyclotron resonance signal plus the continuous background photoconductivity as a function of intensity assumes a maximum and decreases with further increasing intensity. Our measurements show that this effect is of purely kinetic origin controlled by the cyclotron resonance optical transition probability $\sigma_{C R} F$. Additionally we report for the first time on a quantitative comparison between photoconductivity and absorption as functions of intensity. In contrast to the photoconductivity line, the absorption coefficient remains a smooth curve without any indication of a line splitting or even a dip in the line center. At high intensities the line-shape of the absorption coefficient is 
totally different from that of the photoconductivity line. Even at low intensities where the dip in the photoconductivity line does not occur and both line shapes are close to a Lorentzian due to photoionization of shallow donors the linewidths show very different intensity dependences.

The observed phenomena are attributed to optical transitions between $2 p_{-}$and $2 p_{+}$shallow donor levels which are in resonance with $0 \rightarrow 1$ Landau level transitions [3]. Free carriers in the $\mathbf{N}=0$ Landau level are captured into $2 p_{-}$states of ionized donors and subsequently optically excited into $2 p_{+}$levels from where they relax into donor ground states. This mechan ism opens an additional carrier recombination channel which reduces the lifetime of free electrons just at the resonance magnetic field of the $0 \rightarrow 1$ transition and thus decreases the conductivity of the sample. The absorption coeffiecient due to cyclotron resonance is also affected by this optically induced recombination process. The additional absorption caused by $2 p_{-} \rightarrow$ $2 p_{+}$transitions however compensates for the decrease of cyclotron resonance absorption resulting in the observed difference between photoconductivity and the apparent absorption under cyclotron resonance conditions.

Optical transitions between $2 p_{-}$and $2 p_{+}$donor states violate electric dipole selection rules. In compensated samples however electric dipole forbidden transitions, like e.g. the 1 s - $2 \mathrm{~s}$ transition, are regularly observed. They occur due to the intermixing of states having different parity and angular momenta caused by electric stray fields of ionized impurities [4]. This fact explains the sample dependence of the strength of the dip in the photoconductivity line.

Several other mechanisms based on inelastic scattering processes which may yield a reduction of the photosignal or even a dip in the line have also been analyzed. However the drastic difference between the line-shapes of photoconductivity and absorption, and, in particular, the missing of the dip in the absorption line cannot be explained.

We present a detailed calculation in terms of a rate equation model including the above sketched optically controlled recombination process. Using known and plausible kinetic coefficients for thermal and optical transitions rates the experimental results for both photoconductivity and absorption could be reproduced in very good agreement to the measurements.

\section{Experimental}

a) Technique

The measurements were carried out on high-purity n-GaAs epitaxial layers with alloyed ohmic point contacts on opposite edges of 
the samples. The samples were mounted in an immersion cryostate in the center of a superconducting magnet. The laser beam was focused on the sample. Photoconductivity was measured in Faraday configuration with the electric bias field normal to the magnetic field by application of a fast recovery load resistor circuit. In all cases the load resistance was chosen to be much smaller than the sample resistance, thus the relative change of the voltage $V$ across the sample is proportional to the change of the sample conductance, $\Delta V / V \propto$ $\Delta G$. On the other hand, the mobility of n-GaAs at low temperature is not much affected even for large shallow donor ionization rates $[5,6]$, therefore $\Delta G \propto \Delta n$ where $\Delta n$ is the optical generated free electron concentration. The transmission of the epitaxial layers was recorded synchronously to the photoconductive signal by a carbonglass bolometer which was placed just behind the sample.

Cyclotron resonance was excited with several laser lines between $118 \mu \mathrm{m}$ and $570 \mu \mathrm{m}$ using a FIR-molecular-laser pumped by an electrically pulsed low pressure $\mathrm{CO}_{2}$ laser. Measurements will be presented for $\lambda=118 \mu \mathrm{m}$ of $\mathrm{CH}_{3} \mathrm{OH}$ which was the strongest laser line available showing all phenomena most clearly. The FIR laser cavity was tuned and an appropriate laser gas pressure was used to obtain single line and single mode operation. The duration of the laser pulses was $100 \mu \mathrm{s}$, much longer than any expected relaxation time ensuring steady state conditions during optical excitation. The intensity of the laser beam in front of the sample was measured by a calibrated pyroelectric detector and the intensity in the sample was determined by taking into account reflexion losses at the front GaAs surface. Multiple reflexions in the sample were avoided by a wedged piece of the substrate material glued on the back face of the sample. As the laser beam is linearly polarized, the active intensity is one half of the total intensity because in Faraday configuration cyclotron resonance is excited by one circular polarized component of the light.

\section{b) Results and Discussion}

The photoconductive signal and the optical transmission due to cyclotron resonance is shown in Fig. 1 for two different samples and various intensities $I=\hbar \omega F$. The density of donors and compensating acceptors $N_{D}$ and $N_{A}$, respectively, is given at the bottom of the figure. The bias voltage was below the impact ionization instability voltage but above the threshold of photoconductivity onset. The resonance magnetic fields for $0 \rightarrow 1$ and $1 \rightarrow 2$ Landau level transitions, indicated by arrows in Fig. 1, are different due to the nonparabolicity of the conduction band [7]. The photoconductive signal (Fig. 1a and c) shows up on a continuous photoconductivity background due to non-resonant photoionization of shallow donors. At high intensities the apparent splitting of the line growing with rising intensity is clearly observed. The dip in the line occurs just at $0 \rightarrow 1$ Landau level resonance. The line is slightly asymmetric 
because in the high magnetic field wing of the line $1 \rightarrow 2$ transitions occur. The splitting of the line is obviously smaller than the magnetic field separation of $0 \rightarrow 1$ and $1 \rightarrow 2$ transitions for $\lambda=118 \mu \mathrm{m}$ [8] and it is much larger than the spin splitting of Landau levels $[9,10,11]$, which is not resolved in the present measurement. Thus, the cyclotron resonance splitting observed in photoconductivity cannot be attributed to static properties of the conduction band. The strength of the dip is strongly sample

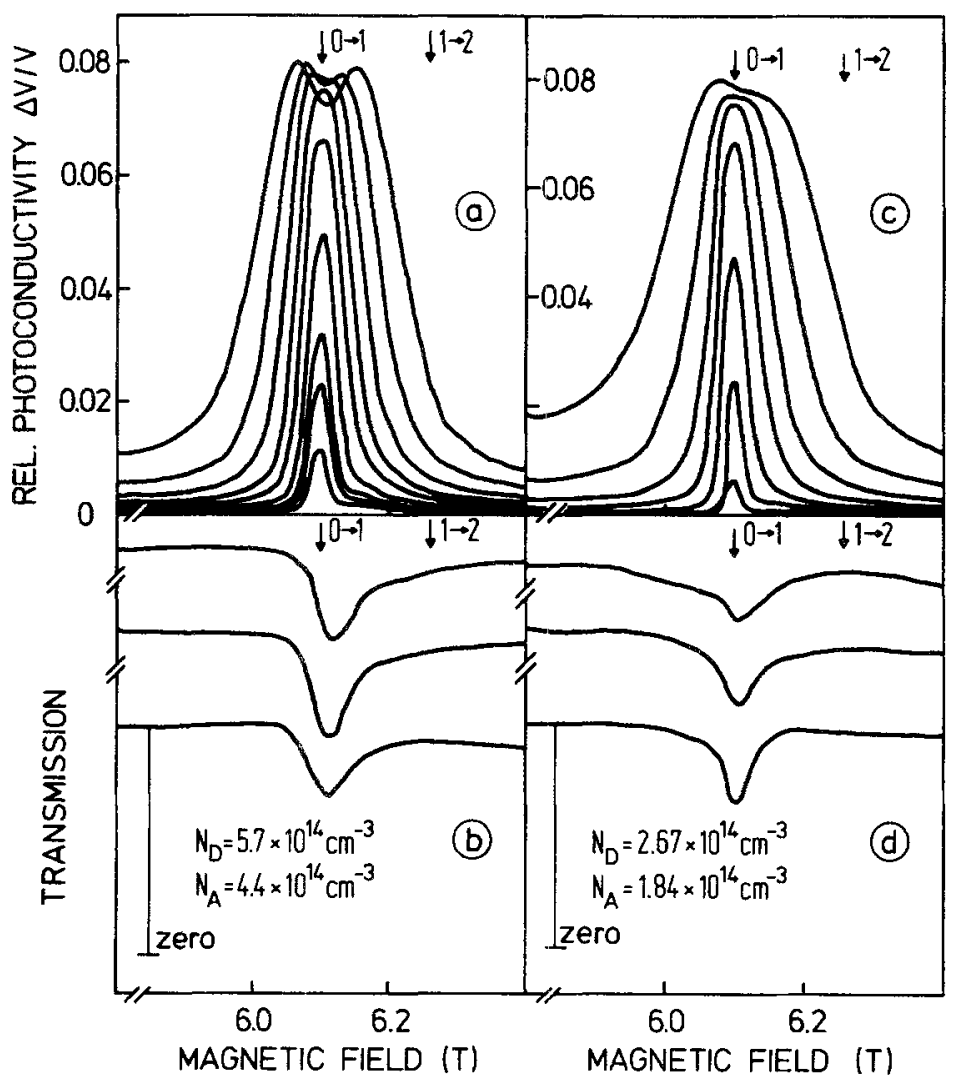

Fig. 1 Cyclotron resonance induced photoconductivity (a and c) in comparison to the optical transmission (b and d) for two different samples. Donor and acceptor densities $\left(N_{D}\right.$ and $\left.N_{A}\right)$ are given at the bottom. Resonance magnetic fields for $0 \rightarrow 1$ and $1 \rightarrow 2$ transitions are indicated by arrows. In (a) and (c) the intensity ratio of subsequent curves from inside to ontside is 1:1.4 with peak intensity $120 \mathrm{~mW} \mathrm{~cm}^{-2}$. The intensities in (b) and (d) are $120 \mathrm{~mW} \mathrm{~cm}-2,44 \mathrm{~mW} \mathrm{~cm}^{-2}, 15 \mathrm{~mW} \mathrm{~cm}^{-2}$ from top to bottom. 
dependent increasing with rising density of ionized impurities at low temperatures $2 N_{A}$. This is demonstrated by the measurements displayed in Fig. 1a in comparison to Fig. 1c. The ionized impurity content of the sample of Fig. 1a is about 2.4 times larger than that of Fig. 1c.

Fig. $1 \mathrm{~b}$ and $\mathrm{d}$ show the optical transmission line as a function of the magnetic field for several intensities. Obviously transmission and therefore the absorption coefficient does not show any splitting or dip for both samples.

In Fig.2 oscilloscope recordings of photoconductivity pulses are shown having been measured in the center of the $0 \rightarrow 1$ Landau level transition for two different intensities. At low intensities the

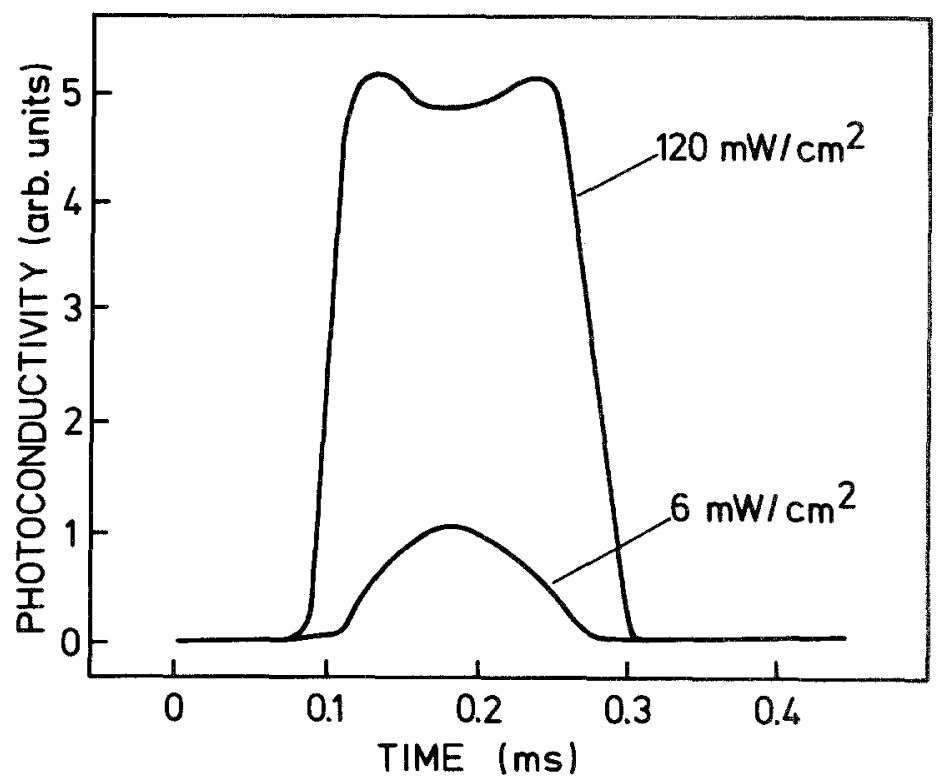

Fig. 2 Recording of the the photosignal pulse in resonance for two different intensities.

recording linearly reproduces the temporal shape of a laser pulse. At high intensities the signal shows a dip just in the middle of the pulse where the intensity is largest. Thus, the dip in photoconductivity is observed either by having constant intensity and tuning the absorption cross section $\sigma_{C R}(B)$ by the magnetic field strength (Fig. 1a) or by keeping $\sigma_{C R}(B)$ constant and varying the intensity. Hence the line splitting is a kinetic effect controlled by the optical transition probability $\sigma_{C R}(B) F$. 
Fig. 3 shows the photoconductive signal $\Delta V / V$ and the absorption coefficient $\alpha$ as functions of intensity obtained at the $0 \rightarrow 1$ transition line center. Both measurements were carried out simultaneously with the same bias voltage on the sample. The absorption coefficient vanishes at zero intensity. Thus thermal generation of free carriers is very small and may be neglected. As the quantum energy of $118 \mu \mathrm{m}$ photons is larger than the binding energy of the 1s donor state, photoionization of shallow donors is the major free carrier generation process below the critical cyclotron resonance transition probability $\left(\sigma_{C R} F\right)_{c}$, smoothing the threshold of photoconductivity onset and yielding an increasing absorption coefficient at low intensities. The intensity dependence of the

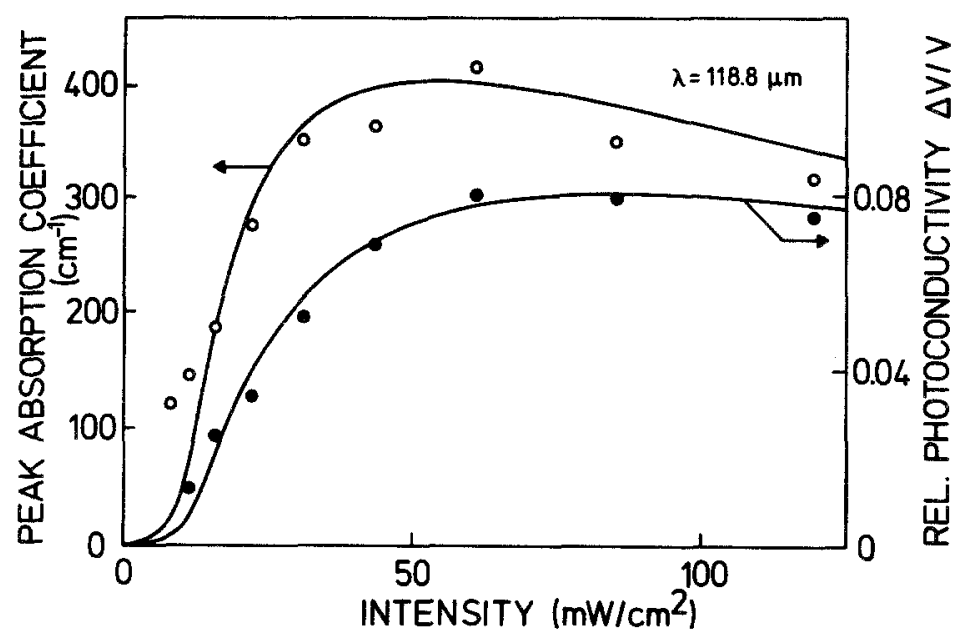

Fig. 3 Absorption coefficient and relative photoconductivity as functions of intensity at the reson ance magnetic field of $N=0$ $\rightarrow \mathrm{N}=1$ Landau level transitions. Full and open circles are measured, lines are calculated.

absorption coefficient $\alpha$ is very similar to that found by G.R. Allan et al. [12] at zero bias voltage in $n$ - GaAs except the fact that in the present case $\alpha$ rises steeper for low intensities due to impact ionization of donors. C.R. Allan et al. attributed the low intensity increase of the absorption coefficient to an intensity dependent generation process which was denoted as impact ionization. In fact their model calculation and the experimental results fit into the present scheme of photoionization. At high intensities $\alpha$ decreases with incrasing intensity due to optical saturation. In this intensity range a constant photosignal is expected, our measurements however clearly 
show that the total photoconductive signal (cyclotron resonance plus background) decreases as displayed in Fig. 3.

In Fig. 4 we finally show the intensity dependence of the observed linewidths demonstrating the totally different behaviour of photoconductivity and absorption. The half width of the pho toconductivity line has been evaluated at half height between the continuous background signal and the signal at the $0 \rightarrow 1$ transition line center. The measurements at highest intensities are somewhat uncertain because of the occurence of $1 \rightarrow 2$ Landau level transitions. At low intensities both half widths are practically equal whereas for high intensities the width of the photoconductivity line grows much faster than that of the absorption line. In both cases the linewidth shows a linear dependence on intensity in contrast to the square root behaviour expected for a homogeneously broadened two-level system.

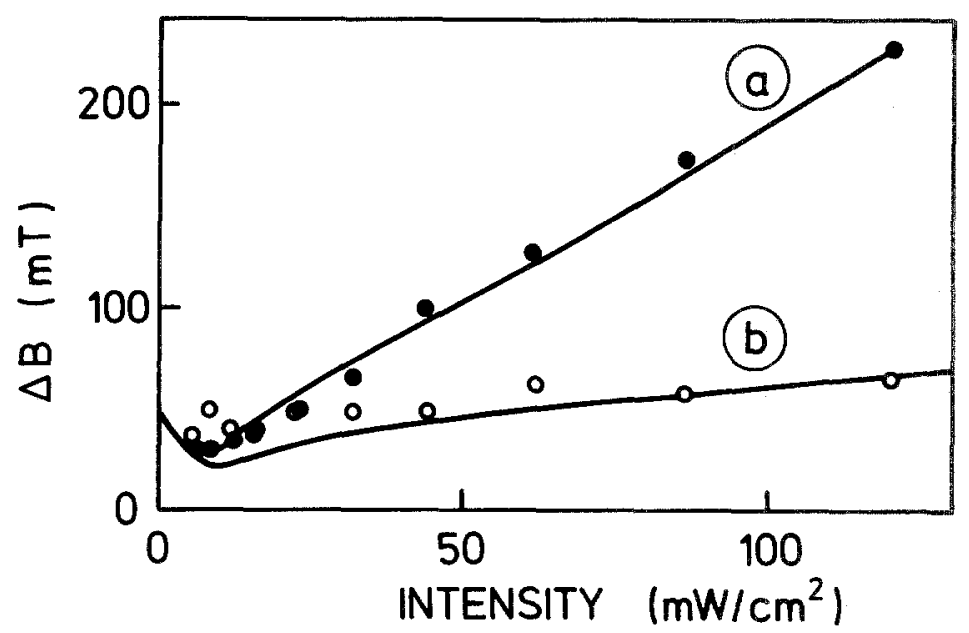

Fig. 4 Linewidth of (a) the photoconductivity line and (b) the absorption line as functions of intensity.

The only conceivable mechanism which explains all observed phenomena is an additional optically induced recombination channel which gives rise to free carriers capture by absorption of photons. Such a mechanism decreases the sample conductance with rising intensity and adds up to the absorption coefficient. This optical process must be in resonance with the $0 \rightarrow 1$ Landau level transition having a similar line width as the unsaturated cyclotron resonance. These requirements are only met by optical transitions between the 
$2 p_{-}$and $2 p_{+}$shallow impurity levels. The energy separations of the $2 p_{+}$and $2 p_{-}$levels, $F_{2 p_{+}}-E_{2 p_{-}}$and the $\mathrm{N}=1$ and $\mathrm{N}=0$ Landau levels, $E_{1}-E_{0}$, are practically equal as can be shown by a simple time inversion argument [3]. In the present case we assume that electrons in the $\mathrm{N}=0$ Landau level are captured in the $2 p_{-}$-shallow donor level, then they are optically excited to the $2 p_{+}$-level and finally they relax to the $1 s$ ground state partly bypassing the $2 p$ level. High power saturation measurements of $1 s-2 p_{+}$transitions showed that a substantial fraction of $2 p_{+}$electrons relax to the donor ground state without cascading across the $2 p_{-}$-level $[5,13]$.

\section{Theory}

\section{a) Model}

To describe the observed cyclotron resonance induced photoconductivity and the absorption under cyclotron resonance conditions we extend the kinetic model used previously to describe the nonlinear photoconductivity [2] by the intensity controlled recombination term discussed above. Additionally we take into account direct photoionization of shallow donors which yields the photoconductivity background smoothing of the photoconductivity threshold and the increase of the absorption coefficient at low intensities. A schematic energy level diagram with arrows indicating all radiative and nonradiative transitions is shown in of Fig. 5. The corresponding rate equations are:

$$
\begin{aligned}
\dot{n}_{1}= & \sigma_{C R} F\left(n_{o}-n_{1}\right)-T_{2}^{S} n_{1} \\
\dot{n}_{0}= & \left(\sigma_{c} F+n X\right) n_{1}+\sigma_{C R} F\left(n_{1}-n_{0}\right)+T_{2}^{S} n_{1} \\
& \left(T_{1}^{S}+T_{3}^{S}\right) n_{0} p_{D}+X_{3}^{S} n_{2 p_{-}} \\
\dot{n}_{2 p_{-}}= & T_{3}^{S} n_{0} p_{D}-X_{3}^{S} n_{2 p_{-}}-\sigma_{i} F\left(n_{2 p_{-}}-n_{2 p_{+}}\right) \\
\dot{n}_{2 p_{+}}= & \sigma_{i} F\left(n_{2 p_{-}}-n_{2 p_{+}}\right)-T_{4}^{S} n_{2 p_{+}}
\end{aligned}
$$

where $n_{0}, n_{1}, n_{12}, n_{2 p_{-}}, n_{2 p_{+}}$and $p_{D}$ are populations of the $\mathrm{N}=0$ and $\mathrm{N}=1$ Landau levels of the $1 s, 2 p$. and $2 p_{+}$donor states and of ionized donors, respectively. The optical cross section of cyclotron resonance, impurity absorption and continuous background absorption are $\sigma_{C R}, \sigma_{i}$ and $\sigma_{c}$, respectively. The coefficient X describes the average impact ionization probability per electron of neutral donors. The parameters $X_{3}^{z}, T_{2}^{z}, T_{3}^{z}$, and $T_{4}^{z}$ denote non-radiative single electron processes whose meaning is obvious from Fig. 5. For the sake of simplicity we assume non-radiative transitions from the $2 p_{+}$level only to the ground state by the rate $T_{4}^{*} n_{2 p+}$ and neglect thermal relaxations of $2 p$ - electrons. All electrons captured into the $2 p$ - state go accross the $2 p_{+}$level to the $1 \mathrm{~s}$ ground state. These simplifications somewhat overemphasize the impurity absorption induced free carrier recombination. 


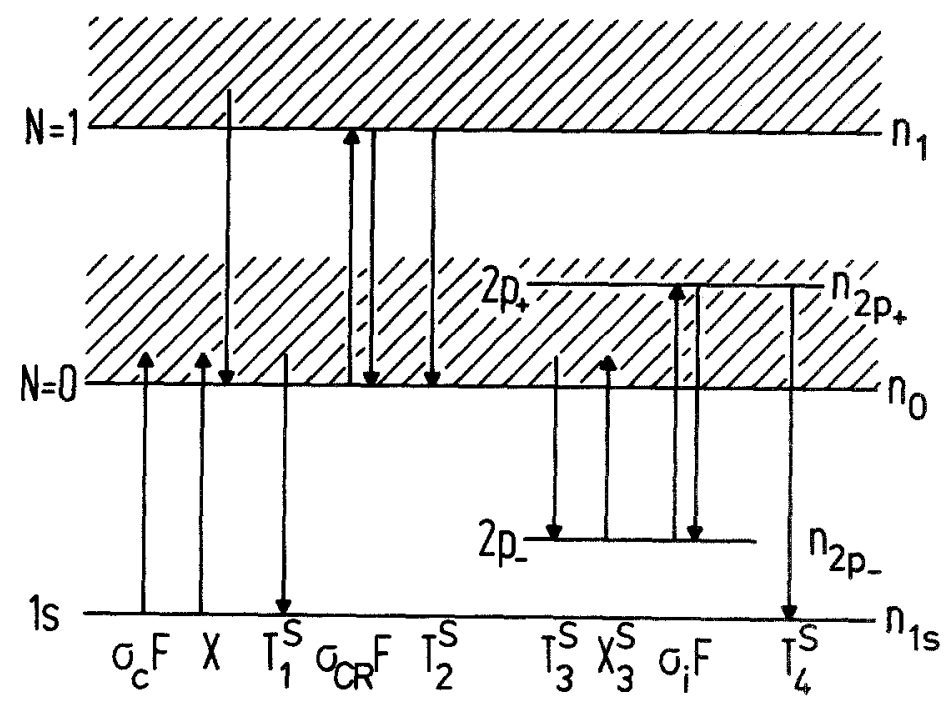

Fig. 5 Schematic energy level diagram and electron generationrecombination processes indicated by arrows. For details see text.

In the steady state $\left(\frac{d}{d t}=0\right)$ and under local neutrality condition, $p_{D}=N_{A}+n$, the free electron concentration $n=n_{0}+n_{1}$ is determined by the balance of carrier generation $g(n, F)$ and recombination $r(n, F)$ :

$$
g(n, F)=r(n, F)
$$

where $N_{A}$ is the density of compensating acceptors.

The rate equation Eq.(1) yields:

$$
g(r, F)=\left(P_{A}-n\right)\left(\sigma_{c} F+n X\right)
$$

with $P_{A}=N_{D}-N_{A}$ the effective donor density and:

$r(n, F)=n\left(N_{A}+n\right) \frac{\sigma_{C R} F+T_{2}^{S}}{2 \sigma_{C R} F+T_{2}^{S}} \times$

$\times\left(T_{1}^{s}+\frac{T_{3}^{S} \sigma_{i} F}{X_{3}^{S}\left(\sigma_{i} F+T_{4}^{S}\right)+\sigma_{i} F T_{4}^{S}}\left[\left(2+\frac{T_{4}^{S}}{\sigma_{i} F}\right)\left(\sigma_{\mathrm{c}} F+n X\right)+T_{4}^{S}\right]\right)$

The absorption coefficient $\alpha=\alpha_{C R}+\alpha_{i}$ of the spectrally coinciding cyclotron resonance and the $2 p_{-} \rightarrow 2 p_{+}$impurity transition is given 
by:

$$
\begin{aligned}
\alpha & =\sigma_{C R}\left(n_{1}-n_{2}\right)+\sigma_{i}\left(n_{2 p_{-}}-n_{2 p_{+}}\right)= \\
& =\sigma_{C R} \frac{n}{1+2 \sigma_{C R} F \tau_{1}}\left(1+\frac{\sigma_{i}}{\sigma_{C R}} \cdot \frac{\left(N_{A}+n\right) T_{3}^{2}\left(1+\sigma_{C R} F \tau_{1}\right)}{\left(1+\frac{\sigma_{i} \bar{F}}{T_{4}^{b}}\right) X_{3}^{*}+\sigma_{i} F}\right)
\end{aligned}
$$

\section{b) Results and Discussion}

The recombination rate $r(n, F)$ in Eq. (4) is of third order in the free electron concentration $n$. The recombination of electrons from the $N=0$ Landau level into the 1s donor ground state is taken into account by a one-step process controlled by the coefficient $T_{1}^{*}$. In fact free electrons are captured in excited donor states and cascade down to the ground state. High power saturation measurements of direct photoionization and photothermal ionization of shallow donors in n-GaAs using the UCSB Free-Electron-Laser and measurements of photocurrent decay after short laser pulse excitation revealed a distinct difference between the capture time of electrons, $\tau_{\text {capture }}$, and the cascade transition time $\tau_{\text {cascade }}[5,13]$. Typically $\tau_{\text {captuse }}=10 \mathrm{~ns}$ was observed whereas $\tau_{\text {carcade }}$ was more than 10 times longer. Thus even for high free carrier generation rates the concentration $\mathbf{n}$ remains significantly smaller than $P_{A}=N_{D}-N_{A}$. The saturated free electron density is about $n_{\infty}=P_{A} \tau_{\text {capture }} /\left(\tau_{\text {captupe }}+\tau_{\text {cascade }}\right) \sim 0.1 P_{A}$. For the present samples which are more than 50 pc compensated (see Fig. 1), $n$ is also much smaller than $N_{A}, n \ll N_{A}$, and we may neglect $\mathrm{n}$ compared to $N_{A}$ in Eq. (4). Within this approximation $\mathrm{Eq} .(2)$ is an easily solvable quadratic equation in $\mathrm{n}$. To simplify calculations we will keep the one step recombination process and denote the transition time from the $N=0$ Landau level to the ground state by $\tau_{1}=\left(T_{1}^{*}\right)^{-1}=\tau_{\text {capiuse }}+\tau_{\text {carcade }}$.

Before evaluating the full range of solutions of Eq. (2) we will discuss several mechanisms which might lead to a suppression of the photosignal or a dip in the photoconductivity line without making use of $2 p_{-} \rightarrow 2 p_{+}$transitions. Conceivable processes are generation of longitudiual optical phonons by electrons scattered into the $\mathrm{N}=2$ Landau level, inelastic scattering of $N=1$ electrons by neutral donors in the $2 p_{-}$state, and laser action between the $2 p_{+}$and $1 s$ donor states

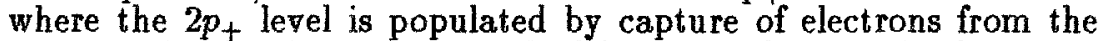
$N=1$ Landau level. In all cases the lifetime of electrons in the upper Landau level $\tau_{2}=\left(T_{2}^{\prime}\right)^{-1}$ is reduced and thus the photoconductive signal is diminished. Emission of LO-phonons is possible because the energy separation between the $\mathrm{N}=2$ Landau level and the donor ground state, $E_{2}-E_{1}$, is slightly larger than the quantum energy of LO-phonons $\hbar \omega_{L O}$. Inelastic collisions of $N=1$ electrons with donors in the $2 p_{-}$state may excite $2 p_{+}$states while the free electrons are scattered into the $\mathrm{N}=0$ Landau level. Though wave vector is 
not conserved in scattering processes on lattice defects, a resonant enhancement of the deexcitation of the $N=1$ Landau level seems to be plausible if the energy separations of the involved Landau levels and impurity states are equal. Thus the lifetime of electrons in the upper Landau level $\tau_{2}$ is resonantly reduced and a dip in the photosignal at line-center may occur. As a threshold phenomenon laser action involving $2 p_{+}$and 1 donor states limits the population of electrons in the conduction band and therefore flattens the top of the photoconductivity line. Experimental search for $2 p_{+} \rightarrow 1 \mathrm{~s}$ laser radiation failed and we do not believe this mechanism being effective in present range of pump intensity.

Neglecting the $2 p_{-} \rightarrow 2 p_{+}$impurity absorption, $\sigma_{i}=0$, we find from Eq.(5): the relation

$$
\alpha=\alpha_{C R}=\sigma_{C R} \frac{n}{1+2 \sigma_{C R} F \tau_{1}}
$$

relating the free electron concentration $n$ to the absorption coefficient $\alpha$ which is now solely due to cyclotron resonance. For the sake of simplicity we ignore photoionization of shallow donors for the following, $\sigma_{c}=0$, which causes only a very small contribution to the photosignal compared to cyclotron resonance (see Fig. 1). In the limit of $n \ll N_{A}$ we obtain the well known solution of Eq. (2) [2].

$$
\begin{aligned}
& n(F)=0 \text { for } \sigma_{C R} F \leq\left(\sigma_{C R} F\right)_{c} \\
& n(F)=\eta P_{A} \tau_{e f f} \frac{\sigma_{C R} F-\left(\sigma_{C R} F\right)_{c}}{1+F / F} \text { for } \sigma_{C R} F>\left(\sigma_{C R} F\right)_{c}
\end{aligned}
$$

exhibiting the threshold behaviour of the optically generated electron density $n(F)$. The optical transition probability at threshold is given by $\left(\sigma_{C R} F\right)_{c}=\tau_{2}^{-1}\left(T_{1}^{*} N_{A}-X P_{A}\right) /\left(2 X P_{A}-T_{1}^{*} N_{A}\right) . F=$ $\left(\sigma_{C R} \tau_{e f f}\right)^{-1}$ is the saturation photon flux density where $\tau_{e f f}=$ $\tau_{2}\left[\left(2 X+T_{1}^{*}\right) /\left(X+T_{1}^{\prime}\right)\right]$ is the average time that free carriers excited into the conduction band by the combined effect of impact ionization and cyclotion resonance require to return to the donor ground state, and $\eta=\left(2 X-T_{1}^{*} N_{A} / P_{A}\right) /\left(2 X+T_{1}^{*}\right)$ is a dimensionless quantum efficiency. The base width of the cyclotron resonance line $\triangle B_{C R l}$ depends on $\mathbf{F}$ as

$$
\Delta B_{C R b}=\Delta B_{C R}\left(\frac{\sigma_{C R o} F}{\left(\sigma_{C R} F\right)_{c}}-1\right)^{1 / 2}
$$

where $\Delta B_{C R}$ is the unsaturated half width and $\sigma_{C R 0}$ ist the peak value of the cross section $\sigma_{C R}(B)$. The photoconductivity line vanishes for $F \leq\left(\sigma_{C R} F\right)_{c} / \sigma_{C R o}$ and thus the base width vanishes too. 


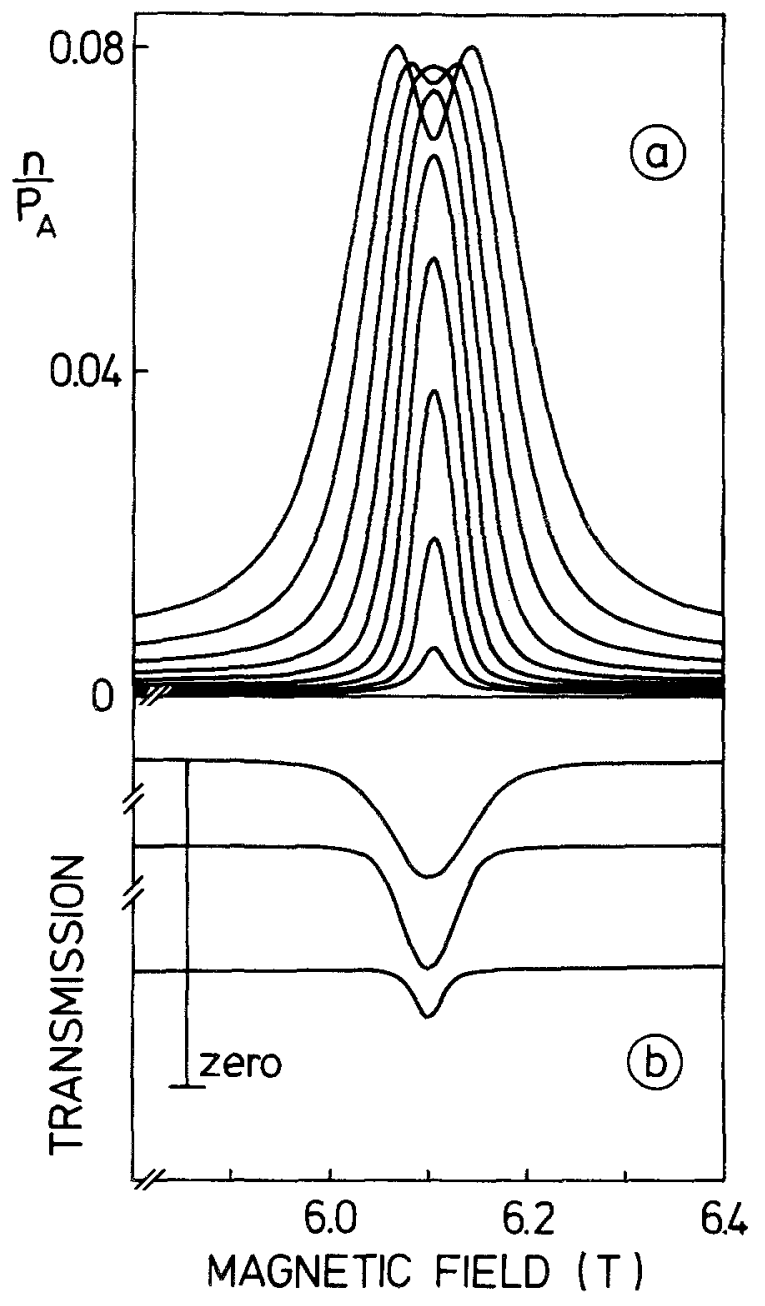

Fig. 6 Calculated photoconductive signal (a) and optical transmission (b) for the experimental conditions of Fig. $1 a$ and $b$. The calculation is based on the parameters: $X P_{A} / T_{1}^{S} N_{A}=$ $0.7, T_{2}^{S} / T_{1}^{S} N_{A}=0.02, X_{3}^{S} / T_{1}^{3} N_{A}=0.5, T_{3}^{S} / T_{1}^{S}=0.002, T_{4}^{S} / T_{1}^{S} N_{A}=$ $0.002, \sigma_{i 0} / \sigma_{C R, 0}=0.5, \sigma_{b} / \sigma_{C R, 0}=2 \cdot 10^{-4}, \Delta B_{C R}=35 \mathrm{mT}$, $\Delta B_{i}=40 \mathrm{mT}$. 
Generally above threshold $n(F) \propto r_{\text {eff }} \propto \tau_{2}$ holds. On the other hand, after Eq.(6) $\alpha$ is proportional to $n(F)$ and thus $\alpha \propto$ $\tau_{2}$. This shows that any spectral structure in the photoconductivity signal caused by a magnetic field dependence of the electron lifetimes $\tau_{2}$ or $\tau_{e f f}$ must be reproduced in the absorption line. Hence lifetime limiting scattering processes, even in resonance with the $2 p_{+}-2 p_{-}$ energy separation, may explain the line shape of photoconductivity however not that of the observed optical transmission.

Because of the interference of cyclotron resonance and impurity absorption and as the absorption coefficient vanishes in the limit $\mathrm{F} \rightarrow 0, \sigma_{C R 0}$ cannot be determined from transmission measurements. Therefore the cross section of $\mathrm{N}=0 \rightarrow \mathrm{N}=1$ transitions was calculated after [14] yielding $\sigma_{C R 0}=4.9 \cdot 10^{-12} \mathrm{~cm}^{2}$. The linewidth $\Delta \omega_{C R}$ was obtained by extrapolating the measurements displayed in Fig. 4 to zero intensity and by evaluating the base width of the photoconductivity line at low intensities according to Eq.(8). In both cases $\Delta B_{C R}=35 m T$ was found. Solutions of Eq.(2) were numerically calculated and the kinetic coefficients were varied to obtain the best fit to the observed photoconductive signal and optical transmission. As the spectral shape and the intensity dependence of both quantities put stringent bounds on the rate constants, a unique set of coefficient was found in spite of the large number of parameters in the fitting procedure. In Fig. 6 the numerically determined photoconductivity and the transmission of the sample are plotted as functions of the magnetic field strength for various intensities corresponding to the measurements of Fig. $1 \mathrm{a}$ and $1 \mathrm{~b}$. The non radiative transition probabilities, the cross sections $\sigma_{i 0}$ and $\sigma_{c}$ and the linewidth $\Delta B_{i}$ expressed in units of $T_{1}^{S} N_{A}, \sigma_{C R o}$, and $\Delta B_{C R}$, respectively, are given in the figure caption. The calculated transmission is in very good quantitative agreement to the measurements shown in Fig. Ib and, by proper choice of the ordinate scale, the calculated photoconductivity curves can be made practically coinciding with the experimental results displayed in arbitrary units in Fig. 1a. The calculated photoconductivity and absorption coefficient at the resonance center and the linewid ths are shown as functions of intensity in Figs. 3 and 4, respectively, as full lines together with the experimental results. Again excellent agreement between measurements and theoretical results is found.

In Figs. 7 and 8 we demonstrate how the impurity absorption induced resonant free electron recombination affects the photoconductivity line and the absorption coefficient. The calculations are carried out for the largest intensity of Fig. 6 corresponding to $F=11 \cdot T_{2}^{\prime} \sigma_{C R 0}^{-1}=0.01 \cdot T_{4}^{z} \sigma_{i 0}^{-1}$. Thus the intensity is significantly higher than the saturation intensity of $0 \rightarrow 1$ Landau level transitions being $T_{2}^{\prime} \cdot\left(\hbar \omega_{C R} \sigma_{C R O}\right)^{-1}$ but still much smaller than the saturation intensity of $2 p_{-} \rightarrow 2 p_{+}$optical excitations, $T_{4}^{*} \cdot\left(\hbar \omega_{C R} \sigma_{i 0}\right)^{-1}$, where 


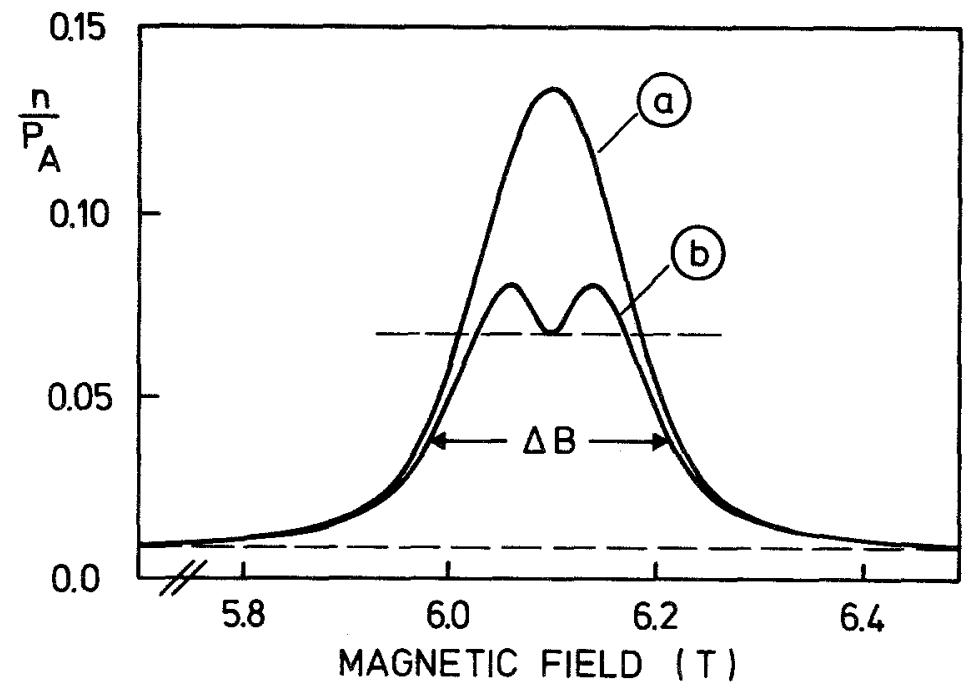

Fig. 7 Calculated photoconductivity line for the parameter set of Fig. 6 and photon flux density $F=11 \cdot T_{2}^{s} \sigma_{C R 0}^{-1}=0.01 \cdot T_{4}^{s} \sigma_{i o}^{-1}$, (a) without shallow donor absorption, $\sigma_{i}=0$, and (b) with shallow donor absorption induced free electron recombination, $\sigma_{i o}=0.5 \sigma_{C R 0}$. Linewid th $\Delta B$ is indicated.

$\hbar \omega_{C R}$ is the cyclotron resonance quantum energy which is equal to $E_{2 p_{+}}-E_{2 p_{-}}$. Figs. 7a and 8a show the photosignal and the absorption coefficient, respectively, without impurity absorption, $\sigma_{i}=0$. Letting $\sigma_{i 0}=0.5 \sigma_{C R 0}$ we find Fig. $7 \mathrm{~b}$ for the photoconductivity line, Fig. $8 \mathrm{~b}$ for the absorption coefficient $\alpha_{C R}$ of the cyclotron resonance alone, and Fig. 8c for the total absorption coefficient under cyclotron resonance conditions $\alpha=\alpha_{C R}+\alpha_{i}$.

Fig. $8 \mathrm{~b}$ corresponds to the situation where a resonant free carrier recombination mechanism is active which, however, does not lead to an additional absorption of photons in reson ance to $\mathrm{N}=0 \rightarrow$ $\mathrm{N}=1$ Landau level transitions. In this case the apparent line splitting is present in both the photosignal and the absorption line (Fig. 7b and $8 \mathrm{~b})$ supporting the conclusions of the above discussion of free carrier lifetime limiting processes which do not involve $2 p_{-} \rightarrow 2 p_{+}$donor transitions. The total absorption coefficient (Fig. 8c) is dominated by the impurity absorption because the Landau level transition is almost saturated. The reason why the photoconductivity line broadens much faster than the absorption line with rising intensity is obvious from Figs. $7 \mathrm{~b}$ and $8 \mathrm{c}$. The intensity dependent free carrier recombination reduces the photoconductive signal in comparison to the case of no- 


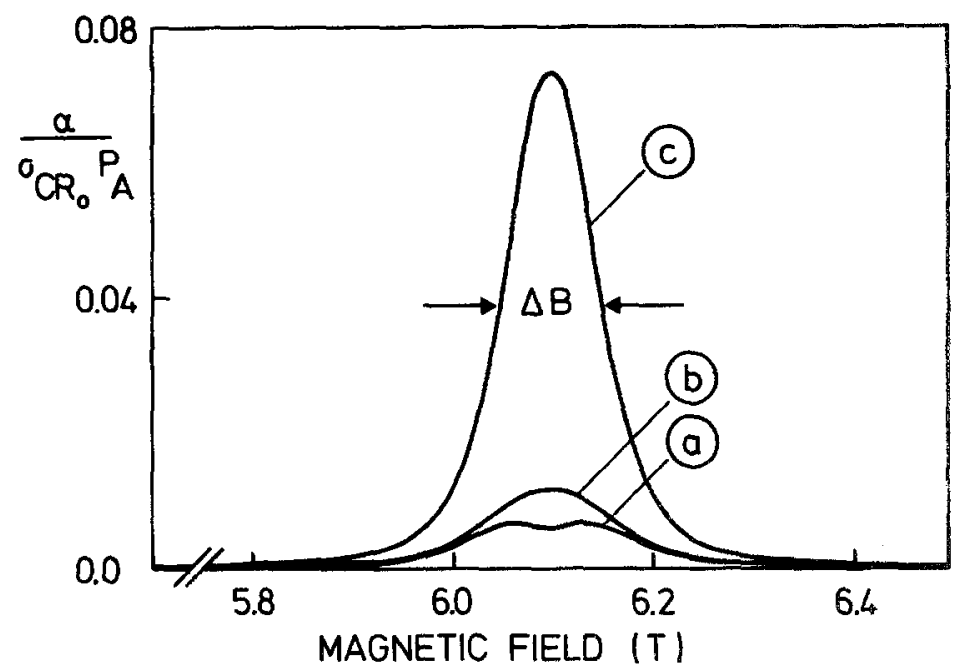

Fig. 8 Calculated absorption line to be compared to the photoconductivity line in Fig. 7. (a) Cyclotron resonance absorption $\alpha=\alpha_{C R}$ without shallow donor transitions, $\sigma_{i}=0$, (b) Cyclotron resonance absorption $\alpha=\alpha_{C R}$ with $\sigma_{i}=0.5 \sigma_{C R 0}$, (c) total absorption under cyclotron resonance condition $\alpha=$ $\alpha_{C R}+\alpha_{i}$ for $\alpha_{i 0}=0.5 \alpha_{C R 0}$. Linewidth $\Delta B$ is indicated.

donor-absorption, $\sigma_{i}=0$ (Fig. 7), and thus increases $\Delta \mathrm{B}$ wheras the donor absorption increases the absorption coefficient (Fig. 8) keeping $\Delta \mathrm{B}$ practically equal to the slowly saturating width of $2 p_{-} \rightarrow 2 p_{+}$ transitions.

From the fitting procedures we essentially obtained the peak absorption cross section $\sigma_{i o}=0.5 \sigma_{C R 0}$, the unsaturated linewidth $\Delta B_{i}=40 \mathrm{mT}$ of the $2 p_{-} \rightarrow 2 p_{+}$donor transition, and the lifetime of electrons in the $N=1$ Landau level $\tau_{2}=\left(T_{2}^{\prime}\right)^{-1}=30 \mathrm{~ns} . \sigma_{i 0}$ is about 5 times the optical absorption cross section of $1 \mathrm{~s} \rightarrow 2 \mathrm{~s}$ shallow donor transitions being likewise electrical dipole forbidden. This seems to be quite large, we have to keep in mind however that the model calculations were simplified by omitting relaxation

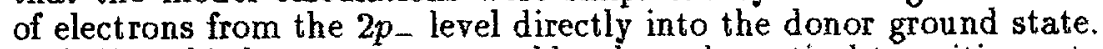
Including this latter process would reduce the optical transition rate inside the donor system being necessary to describe the observed phenomena. The experimental data, however, do not allow to fix a unique branching rate of optical $2 p_{-} \rightarrow 2 p_{+}$and non-radiative $2 p_{-} \rightarrow 1 s$ transitions. On the other hand, the lifetime $\tau_{2}$ depends only on the gross free carrier recombination rate being unaffected by the details of the recombination process. Thus $\tau_{2}$ is a properly 
measured quantity which can only be determined by evaluating the intensity dependence of both the photoconductive signal and the absorption coefficient in the framework of a kinetic model which takes into account an optically induced acceleration of free carrier recombination by absorbing photons in resonance with $0 \rightarrow 1$ Landau level transitions. Neglecting the shallow donor absorption simulates a too large cyclotron resonance absorption coefficient yielding a too small lifetime of electrons in the $N=1$ Landau level. In the present case $\tau_{2}$ turns out to be about 15 times larger than it would be without the impurity transition.

\section{Conclusion}

In summary our results show that photoconductivity and optical absorption under cyclotron resonance conditions in high purity n-GaAs are more complex than it has been assurned previously. As cyclotron resonance can only be measured with non zero free electron concentration, and because the $2 p_{-}$shallow donor level remains below the $\mathrm{N}=0 \mathrm{~L}$ andau level for all magnetic field strengths, a certain population of $2 p_{\text {_ }}$ states must be present and thus the interference between both absorption processes is unavoidable as long as $2 p_{-} \rightarrow 2 p_{+}$electric dipole transitions are activated by ionized impuritics. This interference gets most drastically manifest at high intensities causing an apparent splitting of the photoconductivity line. Even at lower intensities however when the dip in the photoconductivity line is not observable, all optical characteristics previously attributed to cyclotron resonance are affected by the shallow donor absorption. The energy separation $E_{2 p_{+}}-E_{2 p-}$ of shallow donors in a magnetic field is exactly equal to $\hbar w_{C R}$ only for isolated impurities in the effective mass approximation. The same electric stray field of ionized impurities which cause the activation of $2 p_{-} \rightarrow 2 p_{+}$absorption may shift the donor energy levels due to stark effect. For magnetic field strengths $B<1 \mathrm{~T}$ a field dependent deviation of $E_{2 p_{+}}-E_{1 p_{-}}$from the cyclotron resonance quantum energy was observed [15] amounting up to $8 \mathrm{pc}$. In this case we expect that the peak positions of photoconductivity and absorption do not spectrally coincide and do not occur at the resonance magnetic field strength of $0 \rightarrow 1$ Landau level transitions. Thus effective masses determined by standard cyclotron resonance methods at low magnetic fields may be incorrect by a few percent.

Acknowledgements - We thank E. Schöll for many helpful discussions and $\mathbf{E}$. Bauser for provision of the samples. Financial support by the Deutsche Forschungsgemeinschaft is gratefully acknowledged. 


\section{References}

[1] R. Obermaier, W. Böhm, W. Prettl and P. Dirnhofer, Phys. Lett. 105A, 149 (1984).

[2] M. Weispfenning, I. Hoeser, W. Böhm, W. Prettl and E. Schöll, Phys. Rev. Lett. 55, 754 (1985).

[3] H.P. Wagner and W. Prettl, Solid State Commun. 66, 367 (1988).

[4] G.E. Stillman, C.M. Wolfe and J.O. Dimmock, in Semiconductor and Semimetals, vol.12, pp. 169, eds. R.K. Willardson, Academic Press, N.Y. 1977.

[5] J. Kaminski, J. Spector, W. Prettl and M. Weispfenning, Appl. Phys. Lett. 52, 233 (1988).

[6] J.A. Bluyssen, I.C. Maan, T.B. Tan and P. Wyder, Phys. Rev. B 22, 749 (1980).

[7] M. Braun and U. Rössler, J. Phys C18, 3365 (1983)

[8] G. Lindemann, R. Lassnig, W. Seidenbusch and E. Gornik, Phys. Rev. B 28, 4693 (1983).

[9] H. Fetterman, J. Waldman and C.M. Wolfe, Solid State Commun. 11, 375 (1972); H. Sigg, P. Wyder and J.A.A.J. Perenboom, Phys. Rev. B 31, 5253 (1985);

[10] H. Sigg, H.J.A. Bluyssen and P. Wyder, Solid State Commun. 48, $897(1983)$.

[11] V.G. Golubev, V.I. Ivanov-Omskii, I.G. Minervin, A.V. Osutin and D.G. Polyakov, Sov. Phys. JETP 61, 1214 (1985).

[12] G.R. Allan, A. Black, C.R. Pidgeon, E. Gornik, W. Seidenbusch, and P. Colter, Phys. Rev. B 31, 3550 (1978)

[13] J. Kaminski, J. Spector, W. Prettl, and M. Weispfenning, Int. J. Infrared and Millimeter Waves 9, 745 (1988)

[14] K.L. Kobayashi and E. Otsuka, J. Phys. Chem. Solids 35, 839 (1974)

[15] G.E. Stillman, D.M. Larsen, and C.M. Wolfe, Phys. Rev. Letters 27, 989 (1971) 